\title{
Vinyl Carbamate
}

National Cancer Institute

\section{Source}

National Cancer Institute. Vinyl Carbamate. NCI Thesaurus. Code C29864.

A synthetic org anic compound produced in industry, Vinyl Carbamate is used in biomedical research to induce experimental (especially skin and lung) tumors in animals.

Metabolism of Vinyl Carbamate leads to formation of electrophilic reactants that covalently bind to macromolecules (DNA-, RNA-, and protein-adducts) and initiate carcinogenesis. ( $\mathrm{NCl04)}$ 